\title{
Communication
}

\section{Broadband Analysis of Multiscale Electromagnetic Problems: Novel Incomplete-Leaf MLFMA for Potential Integral Equations}

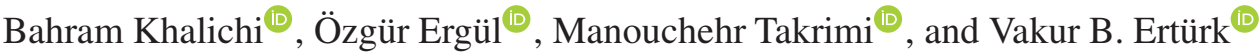

\begin{abstract}
Recently introduced incomplete tree structures for the magnetic-field integral equation are modified and used in conjunction with the mixed-form multilevel fast multipole algorithm (MLFMA) to employ a novel broadband incomplete-leaf MLFMA (IL-MLFMA) to the solution of potential integral equations (PIEs) for scattering/radiation from multiscale open and closed surfaces. This population-based algorithm deploys a nonuniform clustering that enables to use deep levels safely and, when necessary, without compromising the accuracy resulting in an improved efficiency and a significant reduction for the memory requirements (order of magnitudes), while the error is controllable. The superiority of the algorithm is demonstrated in several canonical and real-life multiscale geometries.
\end{abstract}

Index Terms-Incomplete tree structures, low-frequency breakdown, multilevel fast multipole algorithm (MLFMA), multiscale electromagnetic problems, potential integral equations (PIEs).

\section{INTRODUCTION}

The use of multilevel fast multipole algorithm (MLFMA) for the solution of surface integral equations (SIEs) has been verified to be one of the most promising choices for scattering and radiation problems, due to its $\mathcal{O}(N \log N)$ complexity for $N$ unknowns [1]-[3]. However, when we consider multiscale structures that can be best modeled with highly variable meshes, conventional MLFMA either suffers from inaccuracy or displays a significant inefficiency [4], [5] due to the fact that it is a level-based algorithm that deploys fixed-size boxes in its tree structure. Moreover, errors originating from the breakdown of SIE formulations and MLFMA at low frequencies (LFs) should be treated carefully in the analysis of these structures. All these problems worsen as the multiscale factor [4], [5] increases.

Various studies on SIEs, focusing on the LF breakdown and broadband MLFMA implementations for multiscale structures [6]-[23], are available in the literature. Unfortunately, many of them deploy fixed-size boxes in their tree structures that may prevent accuracy and/or efficiency when multiscale structures with high multiscale factors are of concern.

In this study, incomplete tree structures, originally introduced for the magnetic-field integral equation [4], are modified and used in

Manuscript received October 24, 2020; revised April 1, 2021; accepted May 29, 2021. Date of publication June 24, 2021; date of current version December 16, 2021. This work was supported by the Scientific and Technical Research Council of Turkey (TÜBITAK) under Grant 117E113. (Corresponding author: Bahram Khalichi.)

Bahram Khalichi and Vakur B. Ertürk are with the Department of Electrical and Electronics Engineering, Bilkent University, 06800 Ankara, Turkey (e-mail: bahram@ee.bilkent.edu.tr; vakur@ee.bilkent.edu.tr).

Özgür Ergül is with the Department of Electrical and Electronics Engineering, Middle East Technical University, 06800 Ankara, Turkey (e-mail: ozergul@metu.edu.tr).

Manouchehr Takrimi is with the National Magnetic Resonance Research Center (UMRAM), Bilkent University, 06800 Ankara, Turkey (e-mail: mtakrimi@ bilkent.edu.tr).

Color versions of one or more figures in this communication are available at https://doi.org/10.1109/TAP.2021.3090574

Digital Object Identifier 10.1109/TAP.2021.3090574 conjunction with the mixed-form MLFMA [23]-[25] with the objective to employ a truly novel broadband implementation, referred to as incomplete-leaf MLFMA (IL-MLFMA), to the solution of potential integral equations (PIEs), which are shown to be immune to the LF breakdown [22]-[28]. The resultant IL-MLFMA is population-based and deploys nonuniform clustering that enables the use of deep levels safely and only when necessary. Hence, the error is controllable, efficiency is increased, and memory requirements are significantly improved (order of magnitude) in the accurate analysis of scattering/radiation from multiscale conducting open and closed surfaces.

The rest of this communication is organized as follows: Section II describes the implementation of the proposed novel IL-MLFMA for PIEs. Demonstration of the algorithm in terms of accuracy, efficiency, and memory requirements is provided in Section III through several real-life multiscale geometries. An $e^{-i \omega t}$ time convention, where $\omega=$ $2 \pi f$ with $f$ being the operating frequency, is assumed and suppressed throughout this communication.

\section{IMPLEMENTATIONS}

For a perfect electric conductor (PEC) in free space $\left(\varepsilon_{0}, \mu_{0}\right)$ with a surface $S$ and a normal unit vector $\hat{\boldsymbol{n}}$ on its surface, PIEs can be expressed as relationships between scattered and incident fields by satisfying the boundary conditions on $S$ for the electric scalar potential $\phi$ and the tangential component of the magnetic vector potential $(\hat{\boldsymbol{n}} \times \boldsymbol{A})$ [22]-[28]. The surface electric current density, $\boldsymbol{J}$, and the normal component of the magnetic vector potential, $\Sigma=\hat{\boldsymbol{n}} \cdot \boldsymbol{A}$, are the unknowns expanded with Rao-Wilton-Glisson (RWG) [29] and pulse basis functions [22]-[28], respectively. Using a Galerkin method of moments (MoM) solution for the resulting PIEs, a matrix equation of the form

$$
\left[\begin{array}{cc}
\overline{\boldsymbol{\Gamma}}_{\boldsymbol{J}, \boldsymbol{J}} & \overline{\boldsymbol{\Gamma}}_{\boldsymbol{J}, \sigma} \\
\overline{\boldsymbol{\Gamma}}_{\sigma, \boldsymbol{J}} & k_{0}^{2} \overline{\boldsymbol{\Gamma}}_{\sigma, \sigma}
\end{array}\right]\left[\begin{array}{c}
\zeta / c_{0} \\
\boldsymbol{\psi} / \eta_{0}
\end{array}\right]=-\left[\begin{array}{c}
\boldsymbol{a}_{\mathrm{inc}} / \eta_{0} \\
i k_{0} \varepsilon_{0} \boldsymbol{\varphi}_{\text {inc }}
\end{array}\right]
$$

is obtained. In (1), $k_{0}, \eta_{0}$, and $c_{0}$ are the free-space wavenumber, intrinsic impedance, and wave velocity, respectively, while $\zeta$ and $\psi$ contain the unknown coefficients of RWG and pulse basis functions, respectively. In addition, $\boldsymbol{a}_{\text {inc }}$ contains the discrete tested form of the known incident magnetic vector potential, whereas $\varphi_{\text {inc }}$ contains that of the electric scalar potential. Finally, the matrix entries of each submatrix in (1) are given as

$$
\begin{array}{ll}
{\left[\Gamma_{\boldsymbol{J}, \boldsymbol{J}}\right]_{m, n}=\left\langle\boldsymbol{j}_{m}, g, \boldsymbol{j}_{n}\right\rangle ;} & {\left[\Gamma_{\boldsymbol{J}, \sigma}\right]_{m, n}=\left\langle\nabla \cdot \boldsymbol{j}_{m}, g, \sigma_{n}\right\rangle} \\
{\left[\Gamma_{\sigma, \boldsymbol{J}}\right]_{m, n}=\left\langle\sigma_{m}, g, \nabla^{\prime} \cdot \boldsymbol{j}_{n}\right\rangle ;} & {\left[\Gamma_{\sigma, \sigma}\right]_{m, n}=\left\langle\sigma_{m}, g, \sigma_{n}\right\rangle}
\end{array}
$$

where

$$
\left\langle\boldsymbol{f}(\boldsymbol{r}), g\left(\boldsymbol{r}, \boldsymbol{r}^{\prime}\right), \boldsymbol{h}\left(\boldsymbol{r}^{\prime}\right)\right\rangle=\int_{S} d s \boldsymbol{f}(\boldsymbol{r}) \cdot \int_{S^{\prime}} d s^{\prime} g\left(\boldsymbol{r}, \boldsymbol{r}^{\prime}\right) \boldsymbol{h}\left(\boldsymbol{r}^{\prime}\right)
$$

with $g\left(\boldsymbol{r}, \boldsymbol{r}^{\prime}\right)$ being the free-space Green's function. In (3), $\boldsymbol{f}(\boldsymbol{r})$ and $\boldsymbol{h}(\boldsymbol{r})$ can be replaced by scalar functions. 
The fast and accurate solution of (1) using the conventional MLFMA is not feasible for multiscale problems due to the need of electrically small boxes at deeper levels of the constructed tree structures giving rise to the well-known LF breakdown. As a remedy, the mixed-form MLFMA [22]-[24], as a broadband MLFMA solver, uses nondirective stable plane-wave MLFMA (NSPWMLFMA) at LFs (i.e., box sizes $<\lambda / 8 ; \lambda$ : free-space wavelength). Although stable computations of near- and far-field interactions can be performed via PIEs and the broadband MLFMAs, such as the mixed-form MLFMA, they employ the standard tree structure with fixed-size boxes and regular division of parent boxes into at most eight subboxes and thus offer only the following two inaccurate/inefficient options for multiscale structures modeled with highly nonuniform discretizations:

1) to use an extended number of levels with small leaf-level boxes that would lead to inaccurate interactions since many of these boxes cannot enclose relatively larger discretization elements, especially in the coarsely discretized regions leading to the violation of addition theorem [2] in many times during the far-field calculations;

2) to use a limited number of levels and hence larger leaf-level boxes that would lead to a high computational cost due to $\mathcal{O}\left(N^{2}\right)$ complexity in self- and near-field interactions among these boxes in locally overdiscretized regions.

Therefore, to tackle the abovementioned issues, incomplete tree structures are used in conjunction with the mixed-form MLFMA resulting in a population-based and hence, a nonuniform clustering-based algorithm. Similar to [4] and [5], first a predetermined threshold, called the maximum box population (MBP), is selected to handle such a nonuniform clustering scheme. Then, three different types of boxes are defined for PIEs. The first two types, namely the overcrowded boxes (OCBs) with a population equal to or more than the given threshold and truncated boxes (TBs) with a population less than the given threshold, have already been introduced in [4]. However, because PIEs include both RWG and pulse basis functions, correct organization of near- and far-field interactions as well as their efficient and accurate computations necessitate a third type of boxes called pruned boxes (PBs). A PB is a virtual box, which was supposed to be one of the extensions for a TB. Note that one can define two separate incomplete tree structures; one for RWG and one for pulse basis functions, and implement the algorithm proposed in [4] that does not require PBs. Unfortunately, in such an implementation for PIEs, all near, pseudonear, and far interactions for the submatrices in (1) should be redefined and calculated separately (including four QR decompositions for small boxes), which will significantly increase the complexity of the algorithm especially for $\overline{\boldsymbol{\Gamma}}_{\boldsymbol{J}, \sigma}$ and $\overline{\boldsymbol{\Gamma}}_{\sigma, \boldsymbol{J}}$ submatrices. Hence, we proceed with a novel single incomplete tree structure by selecting the threshold over the RWG functions and implement the algorithm as follows:

1) Identifying the Parent $T B$ of $R W G$ and Pulse (i.e., Triangle) Functions: The incomplete tree structure is constructed based on the MBP of RWG functions, and thus, the parent TBs of RWG functions are identified using the same algorithm presented in [4]. Very often, however, a triangle may fall into a different parent TB from its pair and/or the corresponding RWG function, and its occurrence increases significantly for multiscale geometries at each and every level. Moreover, these different parent TBs can even be located at different levels (usually observed for geometries with large multiscale factors). A simple example for such a scenario is shown in Fig. 1, where three leaf-level TBs (solid lines) and the associated virtual PBs (dashed lines, centers marked with $\times$ ) contain part of an arbitrarily discretized geometry (i.e., RWG and

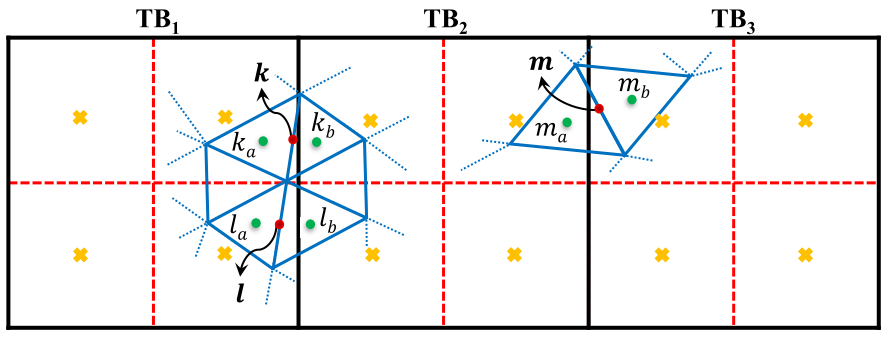

Fig. 1. Pictorial example to illustrate the parent TBs of RWG and pulse basis functions and how their interactions are classified (i.e., near- or far-field interactions).

pulse functions). Consider the RWG $k$ and $l$ together with the associated pair of triangles $\left(k_{a}, k_{b}\right)$ and $\left(l_{a}, l_{b}\right)$, respectively (centroids are marked with dots). It can be clearly seen that $k_{b}$ and $l_{b}$ remain in $\mathrm{TB}_{2}$, while their pair triangles $k_{a}$ and $l_{a}$ as well as the corresponding RWG functions ( $k$ and $l$ ) remain in $\mathrm{TB}_{1}$. To identify the parent $\mathrm{TB}$ of a given triangle and hence the total triangle population in a TB in a robust manner, we first determine its parent $\mathrm{PB}$ by finding the minimum of the shortest distances between the centroid of the triangle and the center of the PBs containing the three edges (i.e., the associated RWGs) of that triangle and then find the parent TB of the corresponding PB. Because this implementation is performed only in the leaf level, it does not bring any extra computational cost (see the time (in seconds) "incomplete tree constructions" in numerical examples).

2) List of Near and Far Boxes: Construction of a list for near boxes, pseudonear boxes, and far boxes are presented in [4].

3) Near- and Far-Field Interactions: All near- and far-field interactions (based on the one-buffer-box criterion) must be calculated for each and every submatrix of (1) (i.e., $\overline{\boldsymbol{\Gamma}}_{\boldsymbol{J}, \boldsymbol{J}}=\overline{\boldsymbol{\Gamma}}_{\boldsymbol{J}, \boldsymbol{J}}^{N}+$ $\overline{\boldsymbol{\Gamma}}_{\boldsymbol{J}, \boldsymbol{J}}^{F}$ (the same for $\overline{\boldsymbol{\Gamma}}_{\boldsymbol{J}, \sigma}, \overline{\boldsymbol{\Gamma}}_{\sigma, \boldsymbol{J}}$ and $\overline{\boldsymbol{\Gamma}}_{\sigma, \sigma}$ ); N: near-field calculation and F: far-field calculation). Yet, the possibility that the parent $\mathrm{TB}$ of a triangle can be different than its pair triangle and/or the corresponding RWG function, as explained in step 1 , should be considered in these calculations. As an example, consider the $k^{\text {th }}$ and the $m^{\text {th }}$ RWG functions in Fig. 1. They are in the far zone of each other and their interaction, $\left\langle\boldsymbol{j}_{k}, g, \boldsymbol{j}_{m}\right\rangle$, must be in $\overline{\boldsymbol{\Gamma}}_{\boldsymbol{J}, \boldsymbol{J}}^{F}$. On the other hand, when we consider the interactions between the pulses on the triangles that form these two RWG functions, $k_{a}$ and $m_{b}$ are in the far zone of each other and their interaction $\left\langle\sigma_{k_{a}}, g, \sigma_{m_{b}}\right\rangle$ must be in $\overline{\boldsymbol{\Gamma}}_{\sigma, \sigma}^{F}$. However, $m_{a}$ is in the near zone of both $k_{a}$ and $k_{b}$, and $m_{b}$ is in the near zone of $k_{b}$. Thus, all $\left\langle\sigma_{k_{a}}, g, \sigma_{m_{a}}\right\rangle,\left\langle\sigma_{k_{b}}, g, \sigma_{m_{a}}\right\rangle$, and $\left\langle\sigma_{k_{b}}, g, \sigma_{m_{b}}\right\rangle$ must be in $\overline{\boldsymbol{\Gamma}}_{\sigma, \sigma}^{N}$. Similarly, when we consider the interactions between these RWG functions and the pulses on the triangles that form these RWG functions, $k_{a}$ and $m_{b}$ are in the far zone of $m$ and $k$, respectively. Hence, $\left\langle\nabla \cdot \boldsymbol{j}_{k}, g, \sigma_{m_{b}}\right\rangle$ and $\left\langle\nabla \cdot \boldsymbol{j}_{m}, g, \sigma_{k_{a}}\right\rangle$ must be in $\overline{\boldsymbol{\Gamma}}_{\boldsymbol{J}, \sigma}^{F}$ (and $\left\langle\sigma_{m_{b}}, g, \nabla^{\prime} \cdot \boldsymbol{j}_{k}\right\rangle$ and $\left\langle\sigma_{k_{a}}, g, \nabla^{\prime} \cdot \boldsymbol{j}_{m}\right\rangle$ must be in $\left.\overline{\boldsymbol{\Gamma}}_{\sigma, \boldsymbol{J}}^{F}\right)$. However, $\left\langle\nabla \cdot \boldsymbol{j}_{k}, g, \sigma_{m_{a}}\right\rangle$ and $\left\langle\nabla \cdot \boldsymbol{j}_{m}, g, \sigma_{k_{b}}\right\rangle$ must be in $\overline{\boldsymbol{\Gamma}}_{\boldsymbol{J}, \sigma}^{N}$ (and $\left\langle\sigma_{m_{a}}, g, \nabla^{\prime} \cdot \boldsymbol{j}_{k}\right\rangle$ and $\left\langle\sigma_{k_{b}}, g, \nabla^{\prime} \cdot \boldsymbol{j}_{m}\right\rangle$ must be in $\left.\overline{\boldsymbol{\Gamma}}_{\sigma, \boldsymbol{J}}^{N}\right)$. After organizing all types of interactions, matrices with high degree of sparsity are calculated for all near-field submatrices in (1).

Regarding the far-field interactions, because NSPWMLFMA is used at the LF regime, algorithms presented in [4] for aggregation, translation, and disaggregation operations should be modified accordingly. However, these operations are similar to those presented in [4] at the high-frequency regime. 
TABLE I

Statistics for SCATtering From a $\lambda$ Diameter Nonuniformly Discretized Sphere With a Multiscale Factor of 100 Illuminated by an $x$-Polarized Uniform Plane Wave Propagating Along the z-Direction at $3 \mathrm{GHz}$. A Different Number of MBPs (Corresponding to the Different Simulation levels) Are Chosen (*All Hyphens “_” Mean That the Solver Does Not CONVERGE)

\begin{tabular}{|c|c|c|c|c|c|c|c|c|c|c|c|c|c|c|c|c|c|}
\hline Maximum box population & 8000 & 6500 & 5000 & 3500 & 2000 & 1000 & 500 & 250 & 100 & 80 & 60 & 50 & 40 & 30 & 20 & 10 & 5 \\
\hline Number of levels & 3 & 5 & 6 & 6 & 7 & 8 & 8 & 9 & 10 & 10 & 10 & 11 & 11 & 11 & 11 & 12 & 13 \\
\hline $\begin{array}{l}\text { Relative RMS error for } \\
\text { PIE/IL-MLFMA (\%) }\end{array}$ & 0.67 & 0.67 & 0.67 & 0.67 & 0.67 & 0.71 & 0.47 & 0.47 & 0.48 & 0.49 & 0.49 & 0.91 & 1.01 & 1.17 & 1.12 & 0.47 & 1.45 \\
\hline $\begin{array}{l}\text { Total run-time for } \\
\text { PIE/IL-MLFMA (s) }\end{array}$ & 9940 & 9940 & 9940 & 9940 & 9940 & 9710 & 6740 & 6610 & 6070 & 5390 & 4170 & 4210 & 4210 & 4370 & 4790 & 6470 & 14540 \\
\hline $\begin{array}{l}\text { Total memory requirement for } \\
\text { PIE/IL-MLFMA (MByte) }\end{array}$ & 7639 & 7639 & 7639 & 7639 & 7639 & 6527 & 2543 & 2158 & 1457 & 1022 & 519 & 439 & 358 & 213 & 129 & 47 & 22 \\
\hline $\begin{array}{c}\text { Relative RMS error for } \\
\text { PIE/mixed-form MLFMA (\%) }\end{array}$ & 0.5 & 0.55 & 2.06 & 2.06 & 100.4 & - $^{*}$ & - & - & - & - & - & - & - & - & - & - & - \\
\hline $\begin{array}{l}\text { Total run-time for } \\
\text { PIE/mixed-form MLFMA (s) }\end{array}$ & 7130 & 6045 & 7742 & 7742 & 41713 & - & - & - & - & - & - & - & - & - & - & - & - \\
\hline $\begin{array}{l}\text { Total memory requirement for } \\
\text { PIE/mixed-form } \\
\text { MLFMA (MByte) }\end{array}$ & 4686 & 3414 & 2525 & 2525 & 1356 & - & - & - & - & - & - & - & - & - & - & - & - \\
\hline
\end{tabular}

\section{NUMERICAL RESULTS}

Numerical results are presented to assess the accuracy, efficiency, and memory requirements of the proposed IL-MLFMA for PIEs (referred to as PIE/IL-MLFMA) for various multiscale geometries. The analytical Mie series solutions, the mixed-form MLFMA for PIEs that uses the conventional tree structure (referred to as PIE/mixed-form MLFMA) [23], the MoM solution for PIEs (referred to as PIE/MoM), and a commercial software Ansoft high-frequency structural simulator (HFSS) [30] are used for comparison purposes. In all simulations, both PIE/IL-MLFMA and PIE/mixed-form MLFMA use the same GMRES iterative solver with an error tolerance of $10^{-4}$ and the same conventional MLFMA/NSPWMLFMA parameters as specified in [23] together with a second-order Gaussian quadrature for the integration over the surfaces of triangles. Moreover, both MLFMA solvers use left and right preconditioners obtained via the incomplete lower upper decomposition of the approximate near-field matrices of their own. However, because the definition of near-field boxes is significantly different for the conventional and incomplete tree structures, the sparsity of these preconditioners also differs for PIE/mixed-form MLFMA and PIE/IL-MLFMA. Finally, all the results are generated in MATLAB. All MLFMA results are obtained on a cluster of Intel Xeon X 5472 processors with $3.00 \mathrm{GHz}$ clock rate and 32 GB RAM, whereas the MoM results are obtained on a cluster of Intel Xeon CLX6230 20-core processors with $2.10 \mathrm{GHz}$ clock rate and 512 GB RAM.

Scattering from a $\lambda$ diameter PEC sphere at $3 \mathrm{GHz}$, illuminated by an $x$-polarized uniform plane wave propagating along the $z$-direction (approaching the sphere from the south pole), is our first numerical example. The sphere is meshed nonuniformly in such a way that it possesses a dense discretization around the south pole (edge size of triangles varies from $\lambda / 2000$ at the south pole to $\lambda / 20$ around the north pole) leading to a total number of 21850 unknowns. The results obtained via the PIE/IL-MLFMA are compared with those of the Mie series solution and the PIE/mixed-form MLFMA. The relative rootmean-square (RMS) error defined for the far zone scattered electric field and given in [4] is used to determine the accuracy.

Table I shows the run times, the relative RMS errors, and the memory requirements of the PIE/IL-MLFMA for this PEC sphere geometry as a function MBP. Note that MBP automatically determines the deepest level attained by PIE/IL-MLFMA. The same performance metrics of PIE/mixed-form MLFMA are also given as a function of level in the same table for comparison purposes. Similar to [4] and [5], the optimal MBP value is between 50 and 250 (for different problems) for the best efficiency, and this value may be changed if the memory requirement is an issue. The memory requirement decreases significantly as the MBP decreases (from 7693 to $22 \mathrm{MB}$ ) in the expense of longer run times for very small MBP values. On the other hand, the error remains almost constant except a slight increase for very small MBP values that may not be preferred from the efficiency point of view. Also, note that the incomplete tree construction time is negligible compared to the total run time.

When we compare the performance of PIE/IL-MLFMA with that of PIE/mixed-form MLFMA, the following are observed. If we use a five-level PIE/mixed-form MLFMA, the relative RMS error is $0.55 \%$ (comparable to that of PIE/IL-MLFMA), the total run time is $6045 \mathrm{~s}$ and it requires $3414 \mathrm{MB}$ memory. If the number of level is increased to six, the relative RMS error increases to $2.06 \%$ and the run time increases to $7742 \mathrm{~s}$ (run time increases as error grows due to convergence problems), while the required memory decreases to $2525 \mathrm{MB}$. A seven-level PIE/mixed-form MLFMA does not work for this problem, as shown in Table I. On the other hand, the relative RMS error of PIE/IL-MLFMA for MBP $=60$ is $0.49 \%$, its run time is $4170 \mathrm{~s}$ and its memory requirement is only $519 \mathrm{MB}$ demonstrating the superiority of PIE/IL-MLFMA. Furthermore, if we sacrifice from the efficiency and reduce MBP to 10, the run time becomes $6470 \mathrm{~s}$, which is comparable to that of a five-level PIE/mixed-form MLFMA, and the relative RMS error remains as $0.47 \%$, but the required memory becomes only $47 \mathrm{MB}$.

The far zone scattered electric field (defined as the electric field intensity multiplied by the distance from the object when the distance goes to infinity) and the normalized near zone electric field of a nonuniformly discretized PEC circular cone, shown in Fig. 2(a) with all details, form the second numerical example. It is illuminated by an $x$-polarized uniform plane wave propagating along the $z$-direction at $1 \mathrm{GHz}$. The circular cone is discretized with 11880 triangles (resulting in 29700 unknowns), where the size of triangles varies from $\lambda / 1000$ at its apex to $\lambda / 15$ at its flat base as shown in Fig. 2(a), where the number of unknowns and the corresponding 

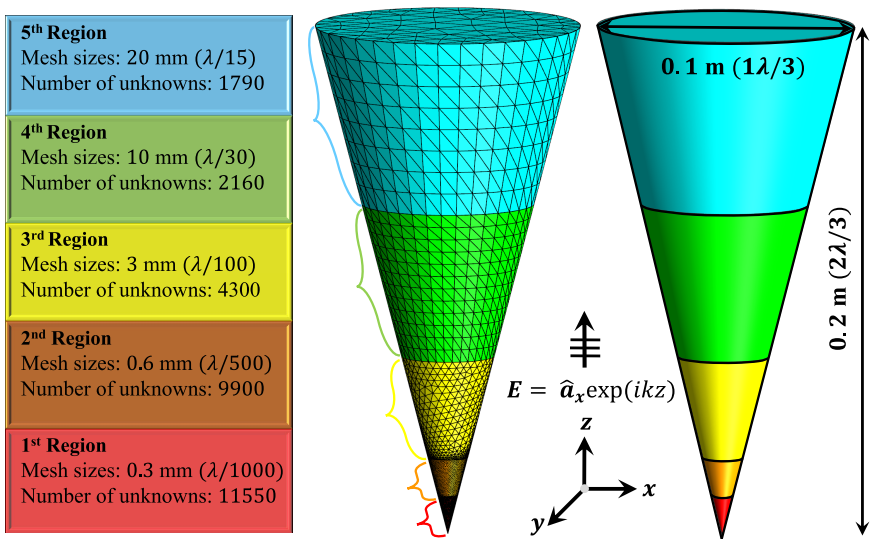

(a)

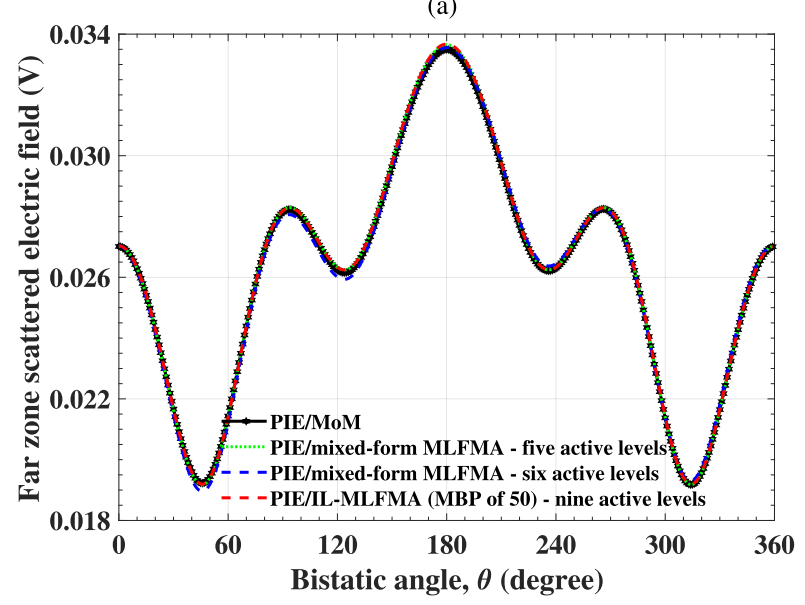

(b)

Fig. 2. (a) Nonuniformly discretized PEC circular cone geometry including the number of unknowns and mesh size for each colored region. (b) Far zone scattered electric-field results obtained via PIE/MoM, PIE/mixed-form MLFMA, and PIE/IL-MLFMA.

mesh size at each region are also highlighted. Fig. 2(b) shows the far zone scattered electric fields as a function of bistatic angle $(\theta)$ on the $x z$ plane $\left(\theta=0^{\circ}\right.$ and $\theta=180^{\circ}$ represent the forward and backward scattering directions, respectively) obtained via PIE/MoM (used as the reference solution), PIE/IL-MLFMA with MBP $=50$, and PIE/mixed-form MLFMAs (five and six levels). The far zone relative RMS error (with respect to MoM) for PIE/IL-MLFMA is $0.42 \%$. This number is $0.44 \%$ for the five-level PIE/mixed-form MLFMA and it increases to $1.08 \%$ if a six-level PIE/mixed-form MLFMA is used. A seven-level PIE/mixed-form MLFMA does not converge. As shown in Fig. 2(b), all results agree well with each other except small discrepancies in the six-level PIE/mixed-form MLFMA results. If we look at the normalized near zone electric fields for this geometry, as shown in Fig. 3(a)-(d) [where the magnitudes of the total electric fields on the $x z$ plane, inside the PEC circular cone as well its vicinity, are illustrated (apex of the cone is assumed to be at the origin)], the near zone relative RMS error for PIE/IL-MLFMA is $0.28 \%$, while it is $0.31 \%$ for the five-level PIE/mixed-form MLFMA. However, this error increases to $11.75 \%$ if the level is increased to six. As shown in Fig. 3(d), the six-level PIE/mixed-form MLFMA suffers from inaccuracies especially at the flat base portion of the cone, where many of the larger basis functions (both RWGs and pulses) stick out from their parent boxes as the box size becomes smaller, especially at the leaf level. On the other hand, regarding the efficiency, PIE/IL-MLFMA is 3.48 times faster and requires nearly

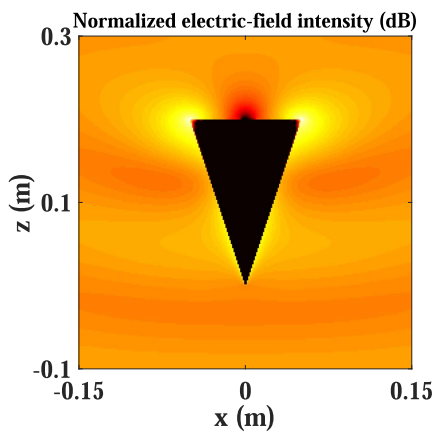

(a)

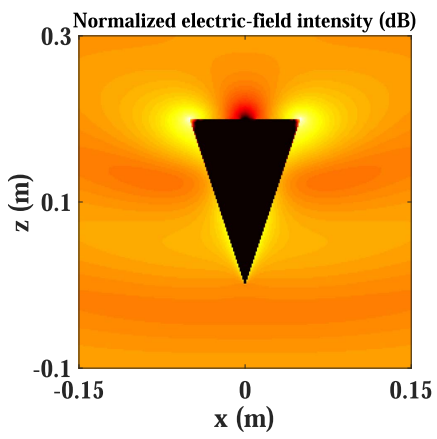

(c)

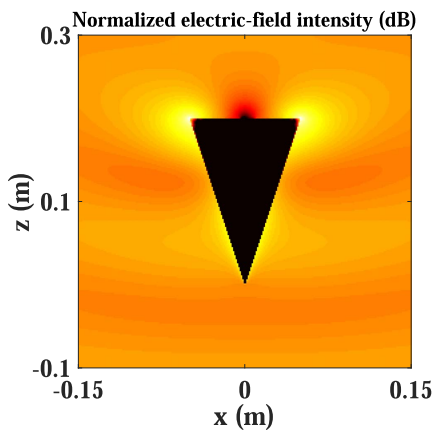

(b)

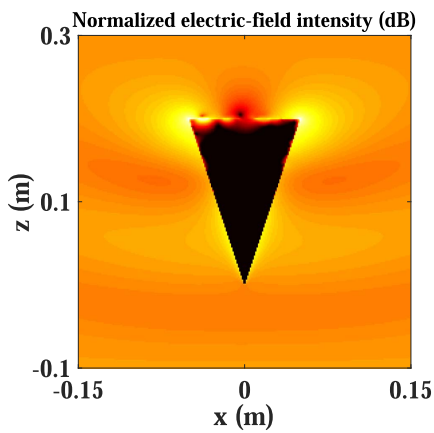

(d)

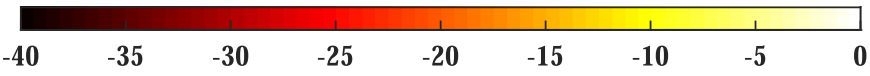

Fig. 3. Normalized electric-field intensities in $\mathrm{dB}$ on the $x z$ plane for the circular cone geometry. Near-field results are obtained via (a) PIE/MoM, (b) PIE/IL-MLFMA with MBP of 50 (corresponding to nine active levels), (c) five-level PIE/mixed-form MLFMA, and (d) six-level PIE/mixed-form MLFMA.

21 times less memory than those of the five-level PIE/mixed-form MLFMA, which is impressive. Furthermore, it is 1.4 times faster and requires 12 times less memory than those of six-level PIE/mixed-form MLFMA despite the big difference in relative RMS errors (both near and far zone errors).

A cavity-backed slot antenna taken from [31] (with the same dimensions except that the dielectric substrate is replaced by air) is the next numerical example. The antenna is excited via a $z$-directed Hertzian dipole, located in the middle of the structure, at $9 \mathrm{GHz}$. The metallic vias and the interdigital capacitor slots on the top surface of the structure are densely meshed $(0.04 \mathrm{~mm})$ and the mesh size is gradually increased ( $2 \mathrm{~mm}$ ) for flat parts, as shown in Fig. 4(a), leading to a total number of 82040 unknowns. Fig. 4(b) and (c) show the absolute value of the normalized current distributions on the top and bottom surfaces, respectively, obtained via PIE/IL-MLFMA with $\mathrm{MBP}=50$. The same type results obtained from HFSS are shown in Fig. 4(d) and (e). Good agreement between these results demonstrates the accuracy of PIE/IL-MLFMA. The radiation pattern of this antenna obtained via both PIE/IL-MLFMA and HFSS also agrees perfectly, as shown in Fig. 4(f).

The analysis of a Helix antenna excited by a voltage source (modeled as a delta-gap generator [1]-[3], [27]) with a feed strength of $1^{v}$ at $500 \mathrm{MHz}$ is our last numerical example. The antenna is nonuniformly discretized as shown in Fig. 5(a), where the size of the triangles varies from $\lambda / 12$ (ground plane) to $\lambda / 600$ (wire and junction parts), resulting in 49676 triangles and 124163 unknowns. As shown in Fig. 5(a), in the zoomed bottom view, a series of RWG functions that create a circular path between the two conductors of 


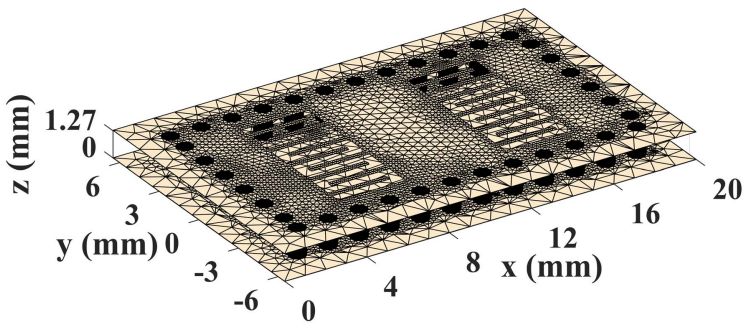

(a)
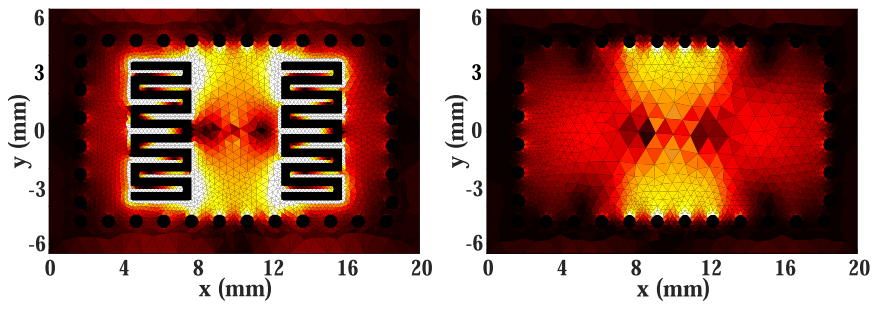

(b)

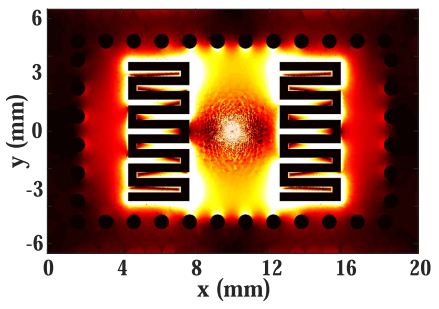

(d)

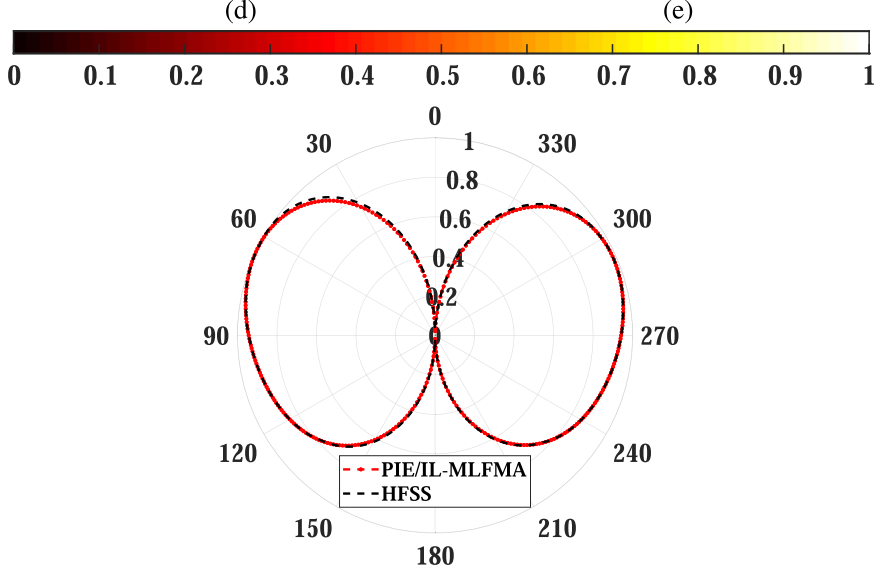

(f)

Fig. 4. (a) Cavity-backed slot antenna excited by a Hertzian dipole located in the middle of the structure. Absolute value of the normalized current distributions on (b) top and (c) bottom surfaces obtained via PIE/IL-MLFMA and the same current distributions on (d) top and (e) bottom surfaces of the antenna obtained by using HFSS at $9 \mathrm{GHz}$. (f) Normalized radiation patterns with respect to $\theta$ when $\varphi=0$ and $\pi$ (i.e., over the $x z$ plane) obtained via PIE/IL-MLFMA and HFSS.

the coaxial cable is considered for applying the delta-gap generators. Fig. 5(b) and (c) show the absolute value of the normalized current distributions in $\mathrm{dB}$ on the surfaces of the antenna obtained via PIE/IL-MLFMA (MBP $=50$ corresponding to eight levels) and HFSS, respectively. Normalized radiation pattern of the antenna with respect to $\theta$ when $\varphi=0$ and $\pi$ (i.e., over the $x z$ plane) obtained via PIE/IL-MLFMA and HFSS are also presented in Fig. 5(d). All in all, the results perfectly agree with each other demonstrating the accuracy of PIE/IL-MLFMA.
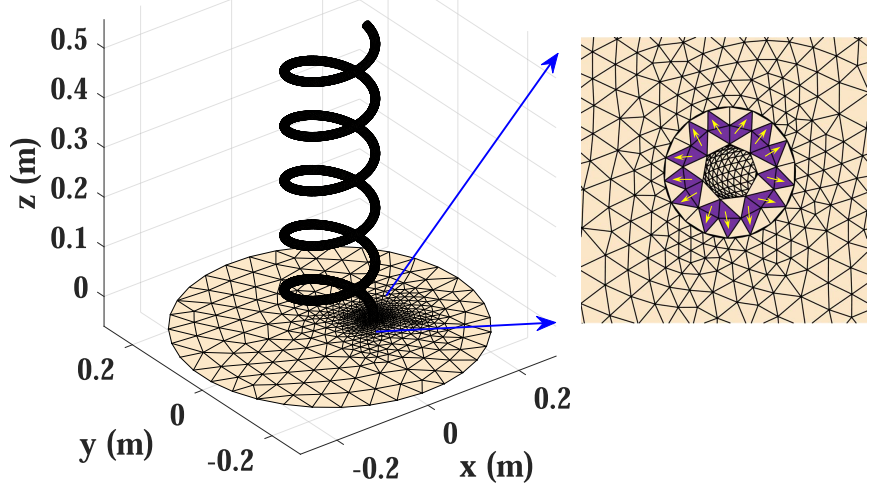

(a)

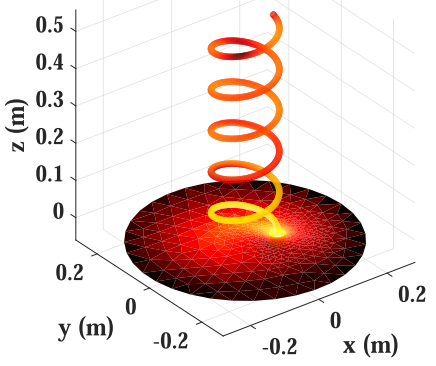

(b)

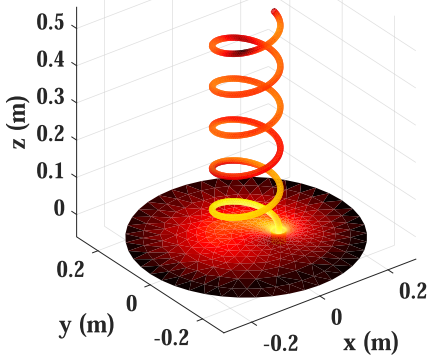

(c)

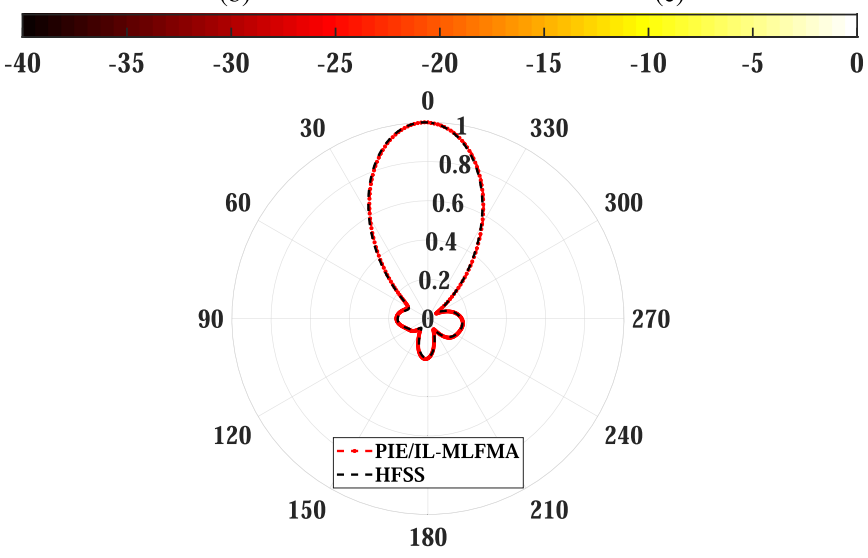

(d)
Fig. 5. (a) 3-D view of a Helix antenna with nonuniform discretization excited by $1^{v}$ circular voltage source over the coaxial line at $500 \mathrm{MHz}$. The zoomed part presents the bottom view and delta-gap excitation used for the Helix antenna. Absolute value of the normalized current distributions in $\mathrm{dB}$ on the surfaces of the antenna obtained via (b) PIE/IL-MLFMA and (c) HFSS (d) Normalized radiation patterns with respect to $\theta$ when $\varphi=0$ and $\pi$ (i.e., over the $x z$ plane) obtained via PIE/IL-MLFMA and HFSS.

\section{CONCLUSION}

Incomplete tree structures are used in conjunction with the mixed-form MLFMA for the solution of PIEs leading to an algorithm that can perform an accurate and efficient analysis of electromagnetic scattering/radiation for multiscale open and closed surfaces. The presented algorithm is population-based and deploys nonuniform clustering for multiscale geometries that are often modeled by using highly variable discretizations. However, it is significantly modified from its predecessor by introducing PBs and reorganizing box-to-box as well as near- and far-field interactions in order to be combined 
with PIEs, which includes both RWG and pulse basis functions. Numerical results obtained from the scattering/radiation analysis of canonical and real-life multiscale geometries clearly demonstrate the superiority of the algorithm. It is accurate, where the error is controllable, and is very efficient in terms of run time and especially memory requirements, where more than an order of magnitude saving in memory can be achieved.

\section{REFERENCES}

[1] W. C. Chew, E. Michielssen, J. Song, and J.-M. Jin, Fast and Efficient Algorithms in Computational Electromagnetics. Norwood, MA, USA: Artech House, 2001.

[2] O. Ergül and L. Gürel, The Multilevel Fast Multipole Algorithm (MLFMA) for Solving Large-Scale Computational Electromagnetic Problems. New York, NY, USA: IEEE Press, 2014.

[3] W. C. Gibson, The Method of Moments in Electromagnetics. Boca Raton, FL, USA: CRC Press, 2007.

[4] M. Takrimi, Ö. Ergül, and V. B. Ertürk, "A novel broadband multilevel fast multipole algorithm with incomplete-leaf tree structures for multiscale electromagnetic problems," IEEE Trans. Antennas Propag., vol. 64, no. 6, pp. 2445-2456, Jun. 2016.

[5] M. Takrimi, O. Ergül, and V. B. Ertürk, "Incomplete-leaf multilevel fast multipole algorithm for multiscale penetrable objects formulated with vol. integral, equations," IEEE Trans. Antennas Propag., vol. 65, no. 9, pp. 4914-4918, Sep. 2017.

[6] O. Ergül and B. Karaosmanoğlu, "Broadband multilevel fast multipole algorithm based on an approximate diagonalization of the Green's function," IEEE Trans. Antennas Propag., vol. 63, no. 7, pp. 3035-3041, Jul. 2015.

[7] I. Bogaert, J. Peeters, and F. Olyslager, "A nondirective plane wave MLFMA stable at low frequencies," IEEE Trans. Antennas Propag., vol. 56, no. 12 , pp. 3752-3767, Dec. 2008.

[8] L. J. Jiang and W. C. Chew, "Low-frequency fast inhomogeneous plane-wave algorithm," Microw. Opt. Technol. Lett., vol. 40, no. 2, pp. 117-122, Jan. 2004.

[9] D. T. Schobert and T. F. Eibert, "Low-frequency surface integral equation solution by multilevel Green's function interpolation with fast Fourier transform acceleration," IEEE Trans. Antennas Propag., vol. 60, no. 3, pp. 1440-1449, Mar. 2012.

[10] J. Zhu, S. Omar, and D. Jiao, "Solution of the electric field integral equation when it breaks down," IEEE Trans. Antennas Propag., vol. 62 , no. 8, pp. 4122-4134, Aug. 2014.

[11] G. Vecchi, "Loop-star decomposition of basis functions in the discretization of the EFIE," IEEE Trans. Antennas Propag., vol. 47, no. 2, pp. 339-346, Feb. 1999.

[12] K. Cools, F. P. Andriulli, F. Olyslager, and E. Michielssen, "Nullspaces of MFIE and CalderÓn preconditioned EFIE operators applied to toroidal surfaces," IEEE Trans. Antennas Propag., vol. 57, no. 10, pp. 3205-3215, Oct. 2009.

[13] Z.-G. Qian and W. C. Chew, "Enhanced A-EFIE with perturbation method," IEEE Trans. Antennas Propag., vol. 58, no. 10, pp. 3256-3264, Oct. 2010.

[14] W.-B. Kong, H.-X. Zhou, W.-D. Li, G. Hua, and W. Hong, "The MLFMA equipped with a hybrid tree structure for the multiscale EM scattering," Int. J. Antennas Propag., vol. 2014, pp. 1-6, Oct. 2014.
[15] X.-M. Pan, J.-G. Wei, Z. Peng, and X.-Q. Sheng, "A fast algorithm for multiscale electromagnetic problems using interpolative decomposition and multilevel fast multipole algorithm," Radio Sci., vol. 47, no. 1, pp. 1-11, Feb. 2012.

[16] J.-G. Wei, Z. Peng, and J.-F. Lee, "Multiscale electromagnetic computations using a hierarchical multilevel fast multipole algorithm," Radio Sci., vol. 49, no. 11, pp. 1022-1040, Nov. 2014.

[17] W.-B. Kong, H.-X. Zhou, K.-L. Zheng, and W. Hong, "Analysis of multiscale problems using the MLFMA with the assistance of the FFT-based method," IEEE Trans. Antennas Propag., vol. 63, no. 9, pp. 4184-4188, Sep. 2015.

[18] J. Carrier, L. Greengard, and V. Rokhlin, "A fast adaptive multipole algorithm for particle simulations," SIAM J. Sci. Stat. Comput., vol. 9, no. 4, pp. 669-686, Jul. 1988.

[19] M. Vikram, H. Huang, B. Shanker, and T. Van, "A novel wideband FMM for fast integral equation solution of multiscale problems in electromagnetics," IEEE Trans. Antennas Propag., vol. 57, no. 7, pp. 2094-2104, Jul. 2009.

[20] S. Hughey, H. M. Aktulga, M. Vikram, M. Lu, B. Shanker, and E. Michielssen, "Parallel wideband MLFMA for analysis of electrically large, nonuniform, multiscale structures," IEEE Trans. Antennas Propag., vol. 67, no. 2, pp. 1094-1107, Feb. 2019.

[21] C. Wang, Z. Jiang, X. Qiao, and T. Wan, "Efficient performance of MLSSM-MLFMA algorithm using adaptive grouping technique for electromagnetic problems," IEEE Trans. Antennas Propag., vol. 66, no. 1, pp. 493-496, Jan. 2018.

[22] B. Khalichi, O. Ergul, M. Takrimi, and V. B. Erturk, "Solution of potential integral equations with NSPWMLFMA," in Proc. IEEE Int. Symp. Antennas Propag. USNC/URSI Nat. Radio Sci. Meeting, Jul. 2018, pp. 2307-2308.

[23] B. Khalichi, O. Ergul, and V. Erturk, "Broadband solutions of potential integral equations with NSPWMLFMA," IEEE Trans. Antennas Propag., vol. 67, no. 6, pp. 4307-4312, Jun. 2019.

[24] B. Khalichi, "Fast and efficient solutions of multiscale electromagnetic problems," Ph.D. dissertation, Dept. Elect. Electron. Eng., Bilkent Univ., Ankara, Turkey, Sep. 2020.

[25] B. Khalichi, V. B. Erturk, and O. Ergul, "Fast solutions of multiscale electromagnetic problems using potential integral equations," in Proc. IEEE Int. Symp. Antennas Propag. North Amer. Radio Sci. Meeting, Jul. 2020, pp. 1071-1072.

[26] W. C. Chew, "Vector potential electromagnetics with generalized gauge for inhomogeneous media: Formulation," Prog. Electromagn. Res., vol. 149, pp. 69-84, 2014.

[27] Q. S. Liu, S. Sun, and W. C. Chew, "A potential-based integral equation method for low-frequency electromagnetic problems," IEEE Trans. Antennas Propag., vol. 66, no. 3, pp. 1413-1426, Mar. 2018.

[28] A. Farshkaran and O. Ergul, "Implementation of the equivalence principle algorithm for potential integral equations," IEEE Trans. Antennas Propag., vol. 67, no. 5, pp. 3249-3255, May 2019.

[29] S. Rao, D. Wilton, and A. Glisson, "Electromagnetic scattering by surfaces of arbitrary shape," IEEE Trans. Antennas Propag., vol. AP-30, no. 3, pp. 409-418, May 1982.

[30] Ansoft HFSS V15.0.0, Ansoft Corp., Pittsburgh, PA, USA, 2012.

[31] Y. Dong and T. Itoh, "Miniaturized substrate integrated waveguide slot antennas based on negative order resonance," IEEE Trans. Antennas Propag., vol. 58, no. 12, pp. 3856-3864, Dec. 2010. 